\title{
THE SECOND DERIVATIVE OF A MEROMORPHIC FUNCTION
}

\author{
J. K. LANGLEY \\ School of Mathematical Sciences, University of Nottingham, \\ Nottingham NG7 2RD, UK (jkl@maths.nott.ac.uk)
}

(Received 19 September 1999)

\begin{abstract}
Let $f$ be meromorphic of finite order in the plane, such that $f^{(k)}$ has finitely many zeros, for some $k \geqslant 2$. The author has conjectured that $f$ then has finitely many poles. In this paper, we strengthen a previous estimate for the frequency of distinct poles of $f$. Further, we show that the conjecture is true if either
\end{abstract}

(i) $f$ has order less than $1+\varepsilon$, for some positive absolute constant $\varepsilon$, or

(ii) $f^{(m)}$, for some $0 \leqslant m<k$, has few zeros away from the real axis.

Keywords: meromorphic function; Nevanlinna theory; zeros of derivatives

AMS 2000 Mathematics subject classification: Primary 30D35

\section{Introduction}

Suppose that $f$ is a function transcendental and meromorphic in the plane. By a theorem of Pólya $[\mathbf{9}, \mathbf{2 6}]$, if $f$ has at least two poles, then for each sufficiently large $k$ the $k$ th derivative $f^{(k)}$ has at least one zero. The following theorem confirmed a conjecture of Hayman [8] from 1959.

Theorem 1.1 (see $[\mathbf{5}, \mathbf{7}, \mathbf{1 8}]$ ). Suppose that $m \geqslant 0$ and $k \geqslant 2$ and that $f$ is meromorphic in the plane such that $f^{(m)}$ and $f^{(m+k)}$ each have finitely many zeros. Then $f^{(m+1)} / f^{(m)}$ is a rational function. In particular, $f$ has finite order and finitely many poles.

We refer the reader to $[\mathbf{2}, \mathbf{6}, \mathbf{1 3}, \mathbf{1 9}, \mathbf{2 3}]$ for related results. Now, Gol'dberg has conjectured that the frequency of distinct poles of $f$ is controlled by the frequency of zeros of a single derivative $f^{(k)}$, provided $k \geqslant 2$, and the author made the following, related conjecture in $[\mathbf{2 1}]$.

Conjecture 1.2. Suppose that $k \geqslant 2$ and $f$ is meromorphic of finite order in the plane and that $f^{(k)}$ has finitely many zeros. Then $f$ has finitely many poles.

Obviously, if Conjecture 1.2 is true for $k=2$, then it is true for $k \geqslant 2$. On the other hand, Conjecture 1.2 is false for functions of infinite order, as shown in [21] by examples of the form $f^{\prime \prime} / f^{\prime}=\mathrm{e}^{h} g^{-1}$ with $g, h$ entire, for which both $f^{\prime}$ and $f^{\prime \prime}$ are zero-free. The 
following theorem, in which the notation is that of $[\mathbf{9}]$, summarizes some results in the direction of Conjecture 1.2.

Theorem 1.3 (see $[\mathbf{2 2}, \mathbf{2 3}]$ ). Suppose that $f$ is meromorphic of finite order $\rho$ in the plane and that $f^{\prime \prime}$ has finitely many zeros. Then

$$
\bar{N}(r, f)=O(\log r)^{3}, \quad r \rightarrow \infty .
$$

If, in addition, $f$ satisfies any one of the following, then $f$ has finitely many poles:

(i) $N\left(r, 1 / f^{\prime}\right)=o\left(r^{1 / 2}\right)$ as $r \rightarrow \infty$;

(ii) $T(r, f)=O\left(r(\log r)^{\delta}\right)$ as $r \rightarrow \infty$, with $\delta$ a constant satisfying $0<3200 \mathrm{e}^{16} \delta<1$;

(iii) there exists $\varepsilon>0$ such that all but finitely many poles $w$ of $f$ have multiplicity $\mu(w) \leqslant|w|^{\rho-\varepsilon}$.

The main results of this paper are substantial improvements of (1.1) and of part (ii) of Theorem 1.3. First we have the following theorem.

Theorem 1.4. Suppose that $f$ is meromorphic of finite order $\rho$ in the plane, and that $f^{\prime \prime}$ has finitely many zeros. Then

$$
\bar{N}(r, f) \leqslant \kappa(\log r)^{2}, \quad r \rightarrow \infty,
$$

in which $\kappa$ is a positive constant depending only on the asymptotic values of $f^{\prime}$.

The key to the proof of Theorem 1.4 is a new way, described in $\S 4$, of estimating $f$ on regions where $f^{\prime}$ is close to its finite asymptotic values. Theorem 1.4 leads to the next result, establishing Conjecture 1.2 for functions of order not much greater than 1 .

Theorem 1.5. There exists a constant $\varepsilon$ with $0<\varepsilon<\frac{1}{2}$ such that if $f$ is meromorphic of order less than $1+\varepsilon$ in the plane and $f^{\prime \prime}$ has finitely many zeros, then $f$ has finitely many poles.

Our last result proves Conjecture 1.2 for functions for which some derivative $f^{(m)}$, with $0 \leqslant m<k$, has relatively few zeros away from the real axis.

Theorem 1.6. Suppose that $0 \leqslant m<k$ and $k \geqslant 2$ and that $\phi(r)$ is a positive function tending to 0 as $r \rightarrow \infty$. Suppose further that $f$ is meromorphic of finite order $\rho$ in the plane, and that $f^{(k)}$ has finitely many zeros. Finally, suppose that

$$
\limsup _{r \rightarrow \infty} \frac{\log ^{+} N^{*}\left(r, 1 / f^{(m)}\right)}{\log r}<\rho_{0}<\frac{1}{2-1 / \rho}
$$

in which $N^{*}\left(r, 1 / f^{(m)}\right)$ counts the zeros of $f^{(m)}$ which lie outside the set $\left\{z:\left|\arg z^{2}\right| \leqslant\right.$ $\phi(|z|)\}$. Then $f$ has finitely many poles. 
Note that by part (ii) of Theorem 1.3 we may assume that $\rho \geqslant 1$ in Theorem 1.6. Functions satisfying the hypotheses of Theorem 1.6 abound: for example $f(z)=1-\mathrm{e}^{\mathrm{i} z}$. There is a substantial literature dealing with entire and meromorphic functions $f$, some of whose derivatives have only real zeros $[\mathbf{1 5}, \mathbf{1 6}, \mathbf{2 9}]$. Theorem 1.6 does not really belong to this strand: rather, in addition to improving part (i) of Theorem 1.3, it shows that some extra geometric information on the distribution of zeros of $f^{(m)}$ suffices to prove Conjecture 1.2.

\section{Lemmas needed for the theorems}

Throughout this paper we denote by $B\left(z_{0}, r\right)$ the Euclidean disc $\left\{z:\left|z-z_{0}\right|<r\right\}$, by $S\left(z_{0}, r\right)$ the circle $\left\{z:\left|z-z_{0}\right|=r\right\}$, and by $A\left(z_{0}, R, S\right)$ the open annulus $\{z: R<$ $\left.\left|z-z_{0}\right|<S\right\}$.

Lemma 2.1 (see $[\mathbf{1 7}, \mathbf{2 2}]$ ). Suppose that $h(z)=\sum_{j=1}^{\infty} a_{j} z^{j}$ maps the disc $B(0, s)$ conformally onto a simply connected domain $D$ of finite area $A$. Then, for real $\theta$ and $0<r<s$, the length $L(r, \theta)$ of the image under $h$ of the line segment $z=t \mathrm{e}^{\mathrm{i} \theta}, 0 \leqslant t \leqslant r$, satisfies

$$
L(r, \theta)^{2} \leqslant \frac{A}{\pi} \log \left(\frac{1}{1-r^{2} s^{-2}}\right) .
$$

Lemma 2.2 (see [24]). Suppose that $d \geqslant 1$ and that $F$ is transcendental and meromorphic in the plane with $T(r, f)=O\left(r^{d}\right)$ as $r \rightarrow \infty$. Then there exist arbitrarily small positive $R$ such that $F(z)$ has no multiple points with $|F(z)|=R$ and the length $L(r, R, F)$ of the level curves $|F(z)|=R$ lying in $|z| \leqslant r$ satisfies $L(r, R, F)=O\left(r^{(3+d) / 2}\right)$ as $r \rightarrow \infty$.

Next we require Tsuji's well-known estimate for harmonic measure [30, p. 116].

Lemma 2.3 (see [30]). Let $D$ be a simply connected domain not containing the origin, and let $z_{0}$ lie in $D$. Let $r \neq\left|z_{0}\right|$. Let $\theta(t)$ denote the angular measure of $D \cap S(0, t)$, and let $D_{r}$ be the component of $D \backslash S(0, r)$ which contains $z_{0}$. Then the harmonic measure of $S(0, r)$ with respect to the domain $D_{r}$, evaluated at $z_{0}$, satisfies

$$
\omega\left(z_{0}, S(0, r), D_{r}\right) \leqslant C \exp \left(-\pi \int_{I} \frac{\mathrm{d} t}{t \theta(t)}\right),
$$

in which $C$ is an absolute constant, and $I=\left[2\left|z_{0}\right|, r / 2\right]$ if $r>4\left|z_{0}\right|$, with $I=\left[2 r,\left|z_{0}\right| / 2\right]$ if $4 r<\left|z_{0}\right|$.

Note that (2.1) for $4 r<\left|z_{0}\right|$ is obtained from the same estimate for the case $r>4\left|z_{0}\right|$ by the substitution $\zeta=1 / z$.

Lemma 2.4. Let $0<\rho<10^{-3}$ and let $\Omega=\{z: \rho<|z|<1, \operatorname{Im}(z)>0\}$. Let $F_{0}=\left\{\mathrm{e}^{\mathrm{i} t}: \pi / 3 \leqslant t \leqslant 2 \pi / 3\right\}$. Let $z_{1}$ lie in $\Omega$ with $200 \rho \leqslant\left|z_{1}\right|$. Then

$$
\omega\left(z_{1}, F_{0}, \Omega\right) \geqslant c \rho^{2}\left(\left|z_{1}\right|^{-1}-\left|z_{1}\right|\right) \sin \left(\arg z_{1}\right),
$$

in which $c$ is a positive constant, independent of $\rho$ and $z_{1}$. 
Proof. Let $d_{j}$ denote positive constants, independent of $\rho$ and $z_{1}$, and set $w=\phi(z)=$ $2 \rho(z+1 / z)$. Then $|z|=\rho$ gives $|w|>3 / 2$, so that $\phi(\Omega)$ contains the semi-disc $D_{1}=$ $\{w:|w|<1, \operatorname{Im}(w)<0\}$. Also $\phi\left(F_{0}\right)=G_{0}$ is a subset of $[-4 \rho, 4 \rho]$ of measure $d_{1} \rho$, and $w_{1}=\phi\left(z_{1}\right)$ has $\left|w_{1}\right| \leqslant 1 / 50$.

Let $\psi$ map $D_{1}$ to the unit disc, with $\psi(-\mathrm{i} / 2)=0$. Then the Schwarz reflection principle (or elementary calculation) gives $d_{2} \leqslant\left|\psi^{\prime}(w)\right| \leqslant 1 / d_{2}$ for $w$ in $D_{1} \cap B\left(0, \frac{1}{4}\right)$, and so Poisson's formula leads to (2.2), since

$$
\omega\left(z_{1}, F_{0}, \Omega\right) \geqslant \omega\left(w_{1}, G_{0}, D_{1}\right) \geqslant d_{3} \rho \operatorname{dist}\left\{w_{1}, \partial D_{1}\right\}=d_{3} \rho\left|\operatorname{Im}\left(w_{1}\right)\right| .
$$

Next we recall that for $0<L<\infty$ and a subset $E$ of $(0, \infty)$ the upper logarithmic density of $E$ satisfies

$$
\overline{\log d e n s} E=\limsup _{r \rightarrow \infty} \frac{\int_{[1, r] \cap E} \mathrm{~d} t / t}{\log r}=\overline{\log \operatorname{den} s}\{t: L t \in E\} .
$$

Lemma 2.5. Let $S(r)$ be an unbounded positive non-decreasing function on $\left[r_{0}, \infty\right)$, continuous from the right, of finite order $\rho$. Let $A>1, B>1$. Then

$$
\overline{\operatorname{logdens}} G \leqslant \rho\left(\frac{\log A}{\log B}\right), \quad G=\left\{r \geqslant r_{0}: S(A r) \geqslant B S(r)\right\} .
$$

Lemma 2.5 is stated in $[\mathbf{1 0}]$ for a characteristic function $T(r, F)$, but the proof goes through for $S(r)$. Finally, we require some standard facts from the Wiman-Valiron theory $[\mathbf{1 1}, \mathbf{3 1}]$. Let $F$ be a transcendental entire function. Provided $r$ is normal for $F$, that is provided $r$ lies outside an exceptional set $E$ of finite logarithmic measure, we have, for $z_{0}$ with $\left|z_{0}\right|=r$ and $\left|F\left(z_{0}\right)\right|>(1-o(1)) M(r, F)$,

$$
\frac{F^{\prime}\left(z_{0}\right)}{F\left(z_{0}\right)}=\nu(r) z_{0}^{-1}(1+o(1))
$$

in which $\nu(r)=\nu(r, F)$ is the non-decreasing central index of $F$. Suppose now that $G$ is transcendental and meromorphic in the plane, with finitely many poles $b_{1}, \ldots, b_{q}$, repeated according to multiplicity. Then $F(z)=G(z) \prod_{j=1}^{q}\left(z-b_{j}\right)$ is entire and the estimate (2.4) holds with $F$ replaced by $G$. Thus, with a slight abuse of notation, we may regard $\nu(r, F)$ as the central index of $G$.

\section{Preliminaries}

Suppose that $h$ is transcendental and meromorphic in the plane, and that $h(z)$ tends to the finite complex number $a$ as $z$ tends to infinity along a path $\gamma$. Then the inverse function $h^{-1}$ is said to have a transcendental singularity over $a[\mathbf{3}, \mathbf{2 5}]$. For each positive $t$, a domain $C(t)$ is uniquely determined as that component of the set $C^{\prime}(t)=\{z$ : $|h(z)-a|<t\}$ which contains an unbounded component of the intersection of $C^{\prime}(t)$ with 
the path $\gamma$. Here $C(t) \subseteq C(s)$ if $0<t<s$, and the intersection of all the $C(t), t>0$, is empty.

The singularity of $h^{-1}$ over $a$ corresponding to $\gamma$ is said to be direct if $C(t)$, for some positive $t$, contains finitely many zeros of $h(z)-a$, and indirect otherwise. If the singularity is direct, then $C(t)$, for sufficiently small $t$, contains no zeros of $h(z)-a$. Singularities over $\infty$ are classified analogously.

Theorem 3.1 (see [3]). If the transcendental meromorphic function $h$ has finite order and the inverse function $h^{-1}$ has an indirect transcendental singularity over $a$, then $a$ is a limit point of critical values of $h$, that is, values taken by $h$ at multiple points of $h$.

Consequently, if $h$ is meromorphic of finite order in the plane with finitely many critical values, then all transcendental singularities of $h^{-1}$ are direct and, by the DenjoyCarleman-Ahlfors Theorem $[\mathbf{3}, \mathbf{2 5}]$, the number of direct transcendental singularities of $h^{-1}$ is at most $2 \rho(h)$.

Next we need a modification of some standard facts discussed in [25]. Suppose that $F$ is a transcendental meromorphic function with finitely many asymptotic values $a_{n}$, and with finitely many critical values $b_{n}$. Suppose that $F$ has no asymptotic values in $c_{0} \leqslant|w|<\infty$ and no critical values in $c_{1} \leqslant|w|<\infty$, where $c_{0} \leqslant c_{1}$. Let $V_{0}$ be the domain obtained by deleting from the annulus $A\left(0, c_{0}, \infty\right)$, the half-open line segment

$$
w=\rho \mathrm{e}^{\mathrm{i} \arg b_{n}}, \quad c_{0}<\rho \leqslant c_{1},
$$

for each finite non-zero critical value $b_{n}$ of $F$.

Consider a component $C_{0}$ of the set $F^{-1}\left(V_{0}\right)$, and choose $z_{0} \in C_{0}$ and $v_{0}$ such that $\mathrm{e}^{v_{0}}=w_{0}=F\left(z_{0}\right)$. Then

$$
\phi(v)=\psi\left(\mathrm{e}^{v}\right)=F^{-1}\left(\mathrm{e}^{v}\right),
$$

with $\psi=F^{-1}$ the branch of the inverse function mapping $w_{0}$ to $z_{0}$, extends by continuation to an analytic function on the simply connected domain $U_{0}=\left\{v: \mathrm{e}^{v} \in V_{0}\right\}$.

Further, $\phi$ maps $U_{0}$ into $C_{0}$. Indeed, $\phi\left(U_{0}\right)=C_{0}$, for if $z^{*} \in C_{0}$ we may join $z_{0}$ to $z^{*}$ by a path $\gamma_{1}$ in $C_{0}$ and choose a path $\gamma_{2}$ in $U_{0}$ starting at $v_{0}$ such that $\mathrm{e}^{\gamma_{2}}=F\left(\gamma_{1}\right)$. Then $F\left(\phi\left(\gamma_{2}\right)\right)=F\left(\gamma_{1}\right)$ and so $\phi\left(\gamma_{2}\right)=\gamma_{1}$ by uniqueness of lifts, since both paths start at $z_{0}$.

There are now two possibilities. The first is that the function $\phi$ is univalent on $C_{0}$, so that the image under $\phi$ of $\operatorname{Re}(v)=1+\log c_{1}$ is a simple curve tending to infinity in both directions. Thus, by a standard argument, such as the Phragmén-Lindelöf principle, $\phi(u) \rightarrow \infty$ as $u \rightarrow \infty$ with $\operatorname{Re}(u)>1+\log c_{1}$, and $C_{0}$ is an unbounded simply connected domain containing a path tending to infinity on which $F(z) \rightarrow \infty$.

On the other hand, if $\phi$ is not univalent in $U_{0}$, then the open mapping theorem shows that $\phi$ has period $k 2 \pi \mathrm{i}$, for some minimal positive integer $k$. In this case $\psi_{1}(\zeta)=\psi\left(\zeta^{k}\right)=$ $\phi(k \log \zeta)$ extends to be analytic in $W_{0}=\left\{\zeta: \zeta^{k} \in V_{0}\right\}$, mapping $W_{0}$ univalently onto $C_{0}$. Also, $\psi_{1}(\zeta)$ has a limit as $\zeta \rightarrow \infty$, which must be finite, and so a pole $z_{1}$ of $F$, since $F$ is transcendental, and $F^{1 / k}: C_{0} \cup\left\{z_{1}\right\} \rightarrow W_{0} \cup\{\infty\}$ is univalent.

The same two possibilities occur for any component $C_{1}$ of the set $\left\{z: c_{0}<|F(z)|<\infty\right\}$ such that $C_{1}$ contains no critical point of $F$. 


\section{An estimate on components where the derivative is small}

Lemma 4.1. Suppose that $G$ is a transcendental meromorphic function of finite order $\rho$ and that $G^{\prime}$ has no asymptotic values in $0<|w| \leqslant d_{1}<\infty$, and no critical values in $0<|w| \leqslant d_{2} \leqslant d_{1}$, and that $G^{\prime}$ has finitely many critical points $z$ with $\left|G^{\prime}(z)\right| \leqslant d_{1}$.

Form the domain $V_{0}$ by deleting from the annulus $A\left(0,0, d_{1}\right)$ the half-open line segment

$$
w=s \mathrm{e}^{\mathrm{i} \arg b_{n}}, \quad d_{2} \leqslant s<d_{1},
$$

for each non-zero finite critical value $b_{n}$ of $G^{\prime}$. Let $D$ be a component of the set $\left(G^{\prime}\right)^{-1}\left(V_{0}\right)$ containing a path $\gamma$ on which $G^{\prime}(z) \rightarrow 0$ as $z \rightarrow \infty$.

Let $N$ be an integer with $N>2+\rho$. Choose $d_{3}$ with $0<d_{3}<d_{2}$ such that $\left|G^{\prime}(z)\right|>d_{3}$ on some circle $S(0, \sigma)$ with $1 \leqslant \sigma \leqslant 2$, and let $D_{1}=\left\{z \in D:|z|>\sigma,\left|G^{\prime}(z)\right|<d_{3}\right\}$. Choose $d$ as in Lemma 2.2, with $0<d<d_{3}$, such that the length of the level curves $\left|z^{N} G^{\prime}(z)\right|=d$ lying in $|z| \leqslant r$ is $O\left(r^{2+\rho}\right)$ for all sufficiently large $r$. Define

$$
u(z)= \begin{cases}\log ^{+}\left|\frac{d}{z^{N} G^{\prime}(z)}\right|, & \text { if } z \in D_{1} \\ 0, & \text { otherwise }\end{cases}
$$

Then $u(z)$ is subharmonic in the plane, and $D$ contains finitely many components $W_{j}$ of the set $\{z: u(z)>0\}$, and these are simply connected. Let $z^{*} \in W_{j}$. Then there exists $M^{*}>0$ such that to each $z \in W_{j}$ corresponds a path $\gamma_{z}$ from $z^{*}$ to $z$, lying in the closure of $W_{j}$, with

$$
\int_{\gamma_{z}}\left|t^{\mu} G^{\prime}(t)\right||\mathrm{d} t| \leqslant M^{*}
$$

for each non-negative integer $\mu$ with $N-\mu>2+\rho$.

Finally, there exist positive constants $S_{0}, S_{1}$ depending on $D$ such that for $z$ in $D$ with $|z|>S_{0}$ and $\left|G^{\prime}(z)\right|<\mathrm{e}^{-1} d_{1}$ we have

$$
|G(z)| \leqslant S_{1}+\frac{C\left|z G^{\prime}(z)\right|}{\log \left|d_{1} / G^{\prime}(z)\right|}
$$

in which $C$ is a positive absolute constant, in particular not depending on $d_{1}, d_{2}, G$ or $D$.

Proof. The $W_{j}$ are simply connected by the maximum principle, since $z^{N} G^{\prime}(z) \neq$ $0, \infty$ on $D_{1}$, by the discussion in $\S 3$. Since $G^{\prime}$ has finite order and

$$
B_{0}(r, u) \leqslant 3 m(2 r, u) \leqslant 3 m\left(2 r, 1 / G^{\prime}\right)+O(\log r), \quad r \rightarrow \infty,
$$

in which $B_{0}(r, u)=\sup \left\{u\left(r \mathrm{e}^{\mathrm{i} t}\right): 0 \leqslant t \leqslant 2 \pi\right\}$, the number of $W_{j}$ is finite [12, Chapter 8].

Next, if $z$ is in $W_{j}$, then we join $z^{*}$ to $z$ by a path $\gamma_{z}$ in the closure of $W_{j}$ consisting of part of the ray $\arg t=\arg z^{*}$, part of the circle $|t|=|z|$, and part of the boundary $\partial W_{j}$ of $W_{j}$. Dividing $\partial W_{j}$ into its intersections with annuli $\left\{z: 2^{q-1}<|t| \leqslant 2^{q}\right\}$ we have

$$
\int_{\partial W_{j}}\left|t^{\mu} G^{\prime}(t)\right||\mathrm{d} t| \leqslant \sum_{q=q_{0}}^{\infty} d 2^{(\mu-N)(q-1)} O\left(2^{q(2+\rho)}\right)+O(1) \leqslant M_{\mu}^{*}
$$


and

$$
\int_{\gamma_{z}}\left|t^{\mu} G^{\prime}(t)\right||\mathrm{d} t| \leqslant M_{\mu}^{*}+\int_{\left|z^{*}\right|}^{\infty} d t^{\mu-N} \mathrm{~d} t+2 \pi d|z|^{1+\mu-N}=O(1)
$$

which proves (4.1).

To prove (4.2), fix $z_{0} \in D$. Let $g=G^{\prime}$ and let $\psi=g^{-1}$ be that branch of the inverse function mapping $w_{0}=g\left(z_{0}\right)$ to $z_{0}$. Choose $v_{0}$ such that $\mathrm{e}^{-v_{0}}=w_{0}$ and set

$$
\phi(v)=\psi\left(\mathrm{e}^{-v}\right)=g^{-1}\left(\mathrm{e}^{-v}\right), \quad H=\left\{v: \mathrm{e}^{-v} \in V_{0}\right\} .
$$

Then $H$ is the half-plane $\left\{v: \operatorname{Re}(v)>\log \left(1 / d_{1}\right)\right\}$ with the half-open line segments

$$
L_{n, q}=\left\{s+q 2 \pi \mathrm{i}-\mathrm{i} \arg b_{n}: \log \left(1 / d_{1}\right)<s \leqslant \log \left(1 / d_{2}\right)\right\}, \quad q \in \boldsymbol{Z},
$$

deleted. Further, as in $\S 3, \phi$ is analytic and univalent on $H$ and $\phi(H)=D$, and $D$ is simply connected.

Now suppose that we attempt to analytically continue $\phi$ along one of the line segments $L_{n, q}$. This continuation can only fail if $\phi(v)$ hits a critical point of $g$ and, since $\phi$ is univalent on $H$, this can only happen for finitely many $L_{n, q}$. Hence there exists a constant $R_{0}>0$ (depending on $D$ ) such that $\phi$ extends analytically and univalently to the set

$$
H_{1}=\left\{v: \operatorname{Re}(v)>c_{0},\left|v-c_{0}\right|>R_{0}\right\}, \quad c_{0}=\log \left(1 / d_{1}\right),
$$

with $\phi(v) \neq 0$ on $H_{1}$. Set

$$
H_{2}=\left\{v: \operatorname{Re}(v)>c_{0},\left|v-c_{0}\right|>100 R_{0}\right\} .
$$

Then $\phi\left(H \backslash H_{2}\right)$ is bounded, since $G^{\prime}$ has no asymptotic value in $0<|w| \leqslant d_{1}$. Further, for $v_{1}$ in $H_{2}, \phi$ is univalent on the disc $B\left(v_{1}, \frac{1}{2}\left(\operatorname{Re}\left(v_{1}\right)-c_{0}\right)\right)$.

We apply a logarithmic change of variables as used in $[\mathbf{1}, \mathbf{2}, \mathbf{4}]$ and elsewhere. Since $\phi \neq 0$ on $H_{1}$, we may define an analytic and univalent branch of $\zeta=\log \phi(v)$ on $H_{2}$. By Koebe's one-quarter theorem [27], we thus have

$$
\left|\frac{\mathrm{d} \zeta}{\mathrm{d} v}\right|=\left|\frac{\phi^{\prime}(v)}{\phi(v)}\right| \leqslant \frac{8 \pi}{\operatorname{Re}(v)-c_{0}}<\frac{32}{\operatorname{Re}(v)-c_{0}}
$$

for $v$ in $H_{2}$. Let $v_{1}$ be in $H_{2}$ with

$$
z_{1}=\phi\left(v_{1}\right), \quad v_{1}=Q+\mathrm{i} y, \quad Q=\log \left|\frac{1}{G^{\prime}\left(z_{1}\right)}\right|>c_{0}+1 .
$$

Let $L$ be the line given by $v=s+\mathrm{i} y, s \geqslant Q$. For $s \geqslant Q$, by (4.3),

$$
\left|\frac{\phi^{\prime}(s+\mathrm{i} y)}{\phi(s+\mathrm{i} y)}\right| \leqslant \frac{32}{s-c_{0}}
$$

and so

$$
|\phi(s+\mathrm{i} y)| \leqslant|\phi(Q+\mathrm{i} y)| \exp \left(\int_{Q}^{s} 32\left(t-c_{0}\right)^{-1} \mathrm{~d} t\right)=|\phi(Q+\mathrm{i} y)|\left(s-c_{0}\right)^{32}\left(Q-c_{0}\right)^{-32},
$$


and, recalling (4.3) and (4.4),

$$
\left|\phi^{\prime}(s+\mathrm{i} y)\right| \leqslant\left|z_{1}\right| 32\left(s-c_{0}\right)^{31}\left(Q-c_{0}\right)^{-32} .
$$

Now $\phi(L)$ is unbounded, and we have

$$
\int_{\phi(L)}\left|G^{\prime}(z)\right||\mathrm{d} z|=\int_{L} \exp (-\operatorname{Re}(v))\left|\phi^{\prime}(v)\right||\mathrm{d} v|=\int_{Q}^{\infty} \mathrm{e}^{-s}\left|\phi^{\prime}(s+\mathrm{i} y)\right| \mathrm{d} s .
$$

Thus, (4.6) and integration by parts give

$$
\int_{\phi(L)}\left|G^{\prime}(z)\right||\mathrm{d} z| \leqslant\left|z_{1}\right| \int_{Q}^{\infty} \mathrm{e}^{-s} 32\left(s-c_{0}\right)^{31}\left(Q-c_{0}\right)^{-32} \mathrm{~d} s \leqslant C_{1}\left|z_{1}\right| \mathrm{e}^{-Q}\left(Q-c_{0}\right)^{-1},
$$

in which $C_{1}$ is a positive absolute constant.

Now we assert that for large $s$ we have $z=\phi(s+\mathrm{i} y) \in W_{j}$, for some $j$. By (4.4) and $(4.5)$,

$$
|z| \leqslant\left|z_{1}\right|\left(s-c_{0}\right)^{32}\left(Q-c_{0}\right)^{-32}
$$

and so

$$
s=\log \left|\frac{1}{G^{\prime}(z)}\right| \geqslant c_{0}+\left(Q-c_{0}\right)\left|\frac{z}{z_{1}}\right|^{1 / 32}
$$

so that

$$
\log |z|=o\left(\log \left|\frac{1}{G^{\prime}(z)}\right|\right)
$$

as $s \rightarrow+\infty$. It follows that a sub-path of $\phi(L)$ joins $z_{1}$ to a point in one of the finitely many $W_{j}$. But $G(z)=O(1)$ on $W_{j}$, so that using (4.7) we deduce (4.2), and Lemma 4.1 is proved.

\section{Critical points and asymptotic values}

Suppose now that $F$ is meromorphic of finite order in the plane, such that $F$ has infinitely many poles, but $F^{\prime}$ has finitely many zeros. Then, by Theorem 3.1, $F$ has finitely many asymptotic values, and each corresponds to finitely many direct transcendental singularities $[\mathbf{3}, \mathbf{2 5}]$ of the inverse function.

Let $J$ be a circle or a simple closed polygonal path, such that every finite asymptotic value of $F$ lies on $J$, but is not a vertex of $J$. Then $J$ divides its complement in $\boldsymbol{C}^{*}=$ $C \cup\{\infty\}$ into two simply connected domains $B_{1}$ and $B_{2}$, such that $B_{1}$ is bounded, while $\infty \in B_{2}$. Fix conformal mappings

$$
h_{m}: B_{m} \rightarrow \Delta=B(0,1), \quad m=1,2, \quad h_{2}(\infty)=0 .
$$

By the Schwarz reflection principle, if $I$ is a line segment contained in $J$ and not meeting any vertex of $J$, then for $m=1,2$ there are positive constants $d_{m}$ such that

$$
d_{m} \leqslant\left|h_{m}^{\prime}(w)\right| \leqslant \frac{1}{d_{m}}, \quad w \in I .
$$


Let $J^{\prime}$ be the set of vertices of $J$ and asymptotic values of $F$, and let $J^{\prime \prime}=J \backslash J^{\prime}$. For each component $J^{*}$ of $J^{\prime \prime}$ we choose an $\operatorname{arc} I_{q}$ of $J^{*}$ whose closure does not meet $J^{\prime}$, and for each such $I_{q}$ there are constants $d_{m}$ as in (5.1).

We consider the components of the sets $F^{-1}\left(B_{m}\right)$. This is more complicated than in $[\mathbf{2 2}]$ because of the different way that $J$ was chosen. It is convenient to take a quasiconformal homeomorphism $\psi_{1}$ of the extended plane onto itself such that $\psi_{1}(\infty)=\infty$ and $\psi_{1}\left(B_{1}\right)=\Delta$. There exist a function $g$ meromorphic in the plane and a quasiconformal mapping $\psi$ such that

$$
\psi(\infty)=\infty, \quad \psi_{1} \circ F=g \circ \psi
$$

This $g$ has finitely many asymptotic values, all of modulus 1 , and $g^{\prime}$ has finitely many zeros. By choosing $\psi_{1}$ appropriately, we may assume that 0 is not a critical value of $g$ and that the distinct finite asymptotic and critical values of $g$ have pairwise distinct principal arguments.

Since $g$ may have finite critical values off the unit circle, we choose $c_{1} \in(0,1)$ and $c_{2}>1$ such that $g$ has no critical values in $|w| \leqslant c_{1}$ nor in $c_{2} \leqslant|w|<\infty$. Let $M$ be the union of the line segments

$$
w=s \mathrm{e}^{\mathrm{i} \arg \zeta_{n}}, \quad c_{1} \leqslant s \leqslant c_{2},
$$

in which the $\zeta_{n}$ are the finite critical values of $g$, and let

$$
A_{1}=B(0,1) \backslash M, \quad A_{2}=\{w: 1<|w| \leqslant \infty\} \backslash M .
$$

Then, as in $\S 3$, all components of the sets $g^{-1}\left(A_{j}\right)$ are simply connected. Further, for each component $T$ of $g^{-1}\left(A_{2}\right)$, either $T$ contains just one pole of $g$, or $T$ contains no pole of $g$, but instead a path tending to infinity on which $g(z)$ tends to infinity. Because the inverse function $g^{-1}$ has finitely many singularities, there are only finitely many components $T$ of this second type.

Consider now a pole $z_{1}$ of $g$. Then $z_{1}$ lies in a component $T_{1}$ of the set $g^{-1}\left(A_{2}\right)$. We assert first that if $z_{1}$ is large enough, then $T_{1}$ is unbounded, and to prove this we assume the contrary. Since $g^{\prime}$ has finitely many zeros the closure $T_{2}$ of $T_{1}$ is a bounded component of the set $\{z:|g(z)| \geqslant 1\}$ and, by analytic continuation, $T_{1}$ is a subset of a bounded component $T_{3}$ of the set $\left\{z: g(z) \in C^{*} \backslash A_{1}\right\}$, such that $g^{\prime}(z) \neq 0$ on $T_{3}$. Hence the set $g^{-1}\left(A_{1}\right)$ has a multiply connected component, which is impossible.

Consider next an unbounded component $S$ of $\{z:|g(z)|<1\}$ having no zero of $g^{\prime}$ in its closure in the finite plane. By $\S 3, S$ is simply connected and conformally equivalent under $g$ to the unit disc. There must be at least one path tending to infinity in $S$ on which $g(z)$ tends to one of its finitely many asymptotic values: we call $S$ type I if there is only one such asymptotic value of $g$ approached along a path tending to infinity in $S$, and type II if there are at least two distinct such values. Clearly a type I component $S$ with no zero of $g^{\prime}$ on its boundary $\partial S$ is such that $\partial S$ consists of just one simple analytic curve going to infinity in both directions, and such an $S$ cannot separate the plane. We shall call an unbounded component $S^{\prime}$ of the set $F^{-1}\left(B_{1}\right)$ type I or II if $S=\psi\left(S^{\prime}\right)$ is a type I or II component of $\{z:|g(z)|<1\}$. 
We return now to a pole $z_{1}$ of $g$, of multiplicity $p$, with $\left|z_{1}\right|$ large, lying in a component $T_{1}$ of $g^{-1}\left(A_{2}\right)$. Then $T_{1}$ is unbounded and cannot share a finite boundary point with another component of $g^{-1}\left(A_{2}\right)$. Thus $T_{1}$ is a subset of a component $T_{4}$ of $\{z:|g(z)|>1\}$, such that $\partial T_{4} \subseteq \partial T_{1}$ and such that $g^{\prime}$ has no zeros in the closure of $T_{4}$ in the finite plane. By $\S 3, T_{4}$ is simply connected and $v(z)=g(z)^{-1 / p}$ is conformal on $T_{4}$. Each boundary point of $T_{4}$ is a boundary point of a component of $g^{-1}\left(A_{1}\right)$. Indeed, the boundary of $T_{4}$ consists of finitely many simple level curves $L^{*}$ of $g$ on which $\arg g(z)$ is monotone, each mapped by $g$ onto an open arc of $|w|=1$. Each such arc must form a boundary curve of a type I or type II component of the set $\{z:|g(z)|<1\}$, with type II for at least one $L^{*}$. In particular, $g$ must have at least two distinct finite asymptotic values and so must $F$.

Lemma 5.1. Let $M_{1}>0$ and let $\phi:[0, \infty) \rightarrow[0, \infty)$ be such that $\phi(r) \rightarrow \infty$ as $r \rightarrow \infty$, and let

$$
A(k)=\left\{z: r^{1 / k} \leqslant|z| \leqslant r^{k}\right\}
$$

for large $r$ and for positive integer $k$. Suppose that $A(2)$ contains $N_{1}$ distinct poles $z_{1}, \ldots, z_{N_{1}}$ of $F$, with $N_{1} \geqslant \phi(r)$. Then provided $r$ is large enough, there exist $N \geqslant c_{0} N_{1}$ distinct type II components $E_{j}$ of the set $F^{-1}\left(B_{1}\right)$, each with the property that

$$
L_{j}=\left\{z \in E_{j}:|V(z)|<1-r^{-M_{1}}\right\} \subseteq A(8), \quad V=h_{1} \circ F .
$$

Here $c_{0}$ is a positive constant depending only on the finite asymptotic values of $F$.

Proof. Let $D_{j}$ be the component of $F^{-1}\left(B_{2}\right)$ in which $z_{j}$ lies, and denote by $\theta_{j}(t)$ the angular measure of the intersection of $D_{j}$ with the circle $S(0, t)$. Since $r$ is assumed large the $D_{j}$ are simply connected.

We shall use in this proof $c$ to denote positive constants, not necessarily the same at each occurrence, but depending only on the asymptotic values of $F$, and in particular not on $r$ or $N_{1}$. By the discussion above, we may assume that at least $256 \mathrm{~N}$ of these $D_{j}$, say $D_{1}, \ldots, D_{256 N}$, with $N$ an integer satisfying

$$
N \geqslant c N_{1} \geqslant c \phi(r)
$$

are such that the following is true. There are distinct finite asymptotic values $a_{1}, a_{2}$ of $F$ such that to each $D_{j}$ corresponds a type II component $E_{j}$ of $F^{-1}\left(B_{1}\right)$, the boundaries of $D_{j}$ and $E_{j}$ sharing a component $K_{j}$. Here $K_{j}$ is a simple piecewise smooth curve going to infinity in both directions and mapped by $F$ onto a fixed sub-path $J_{1}$ of the curve $J$, the closure of $J_{1}$ joining $a_{1}$ to $a_{2}$. Since $F$ is univalent on each $E_{j}$, we have $E_{j} \neq E_{k}$ for $1 \leqslant j<k \leqslant 256 N$.

Now each $D_{j}$ meets $|z|>S_{1}$, and at least $64 N$ of the $D_{j}, 1 \leqslant j \leqslant 256 N$, are such that

$$
\int_{2 r^{2}}^{(1 / 2) r^{4}} \frac{\mathrm{d} t}{t \theta_{j}(t)}>c N \log r
$$


since if (5.5) fails for $D_{1}, \ldots, D_{M}$ we have

$$
\begin{gathered}
M^{2} \leqslant\left(\sum_{j=1}^{M} \theta_{j}(t)\right)\left(\sum_{j=1}^{M} 1 / \theta_{j}(t)\right), \\
c N M \log r \geqslant \sum_{j=1}^{M} \int_{2 r^{2}}^{(1 / 2) r^{4}} \frac{\mathrm{d} t}{t \theta_{j}(t)} \geqslant \frac{M^{2}}{\pi} \log (r / 2) .
\end{gathered}
$$

Of these $64 N$ domains $D_{j}$, at least $16 N$ of them, say $D_{1}, \ldots, D_{16 N}$, have

$$
\int_{2 r^{1 / 4}}^{(1 / 2) r^{1 / 2}} \frac{\mathrm{d} t}{t \theta_{j}(t)}>c N \log r
$$

If the closures of at least $16 N$ of the $D_{j}$ satisfying (5.5) fail to meet $\left\{z:|z| \leqslant 2 r^{1 / 4}\right\}$, then we choose $16 N$ of these domains, and (5.6) is obvious, while otherwise we use the same argument as in (5.5).

We now fix a sub-arc $J_{0}$ of $J_{1}$, one of the $\operatorname{arcs} I_{q}$ chosen following (5.1). We write $p_{j}$ for the multiplicity of the pole of $F$ at $z_{j}$, and for $1 \leqslant j \leqslant 16 N$ we define $v_{j}=\left(h_{2} \circ F\right)^{1 / p_{j}}$, so that $v_{j}$ maps $D_{j}$ conformally onto $\Delta$, with $v_{j}\left(z_{j}\right)=0$. The path $K_{j}$ forming the boundary between $D_{j}$ and $E_{j}$ has a sub-path $\lambda_{j}$ mapped onto $J_{0}$ by $F$. As $z$ describes the $\operatorname{arc} \lambda_{j}$, the image $\left(h_{2} \circ F\right)(z)$ describes an arc of the unit circle of length at least $c$, using (5.1), so that $v_{j}(z)$ describes an arc of the unit circle of length at least $c / p_{j} \geqslant c r^{-\rho(F)-1}$. This gives

$$
\omega\left(z_{j}, \lambda_{j}, D_{j}\right) \geqslant c / p_{j} \geqslant c r^{-\rho(F)-1} .
$$

Set $\sigma_{j}=\lambda_{j} \backslash A(4)$. Since $z_{j}$ lies in $A(2)$, Lemma 2.3, (5.5) and (5.6) imply that

$$
\begin{aligned}
\omega\left(z_{j}, \sigma_{j}, D_{j}\right) & \leqslant c \exp \left(-\pi \int_{2 r^{2}}^{(1 / 2) r^{4}} \frac{\mathrm{d} t}{t \theta_{j}(t)}\right)+c \exp \left(-\pi \int_{2 r^{1 / 4}}^{(1 / 2) r^{1 / 2}} \frac{\mathrm{d} t}{t \theta_{j}(t)}\right) \\
& \leqslant c \exp (-c N \log r) .
\end{aligned}
$$

Thus (5.4) and (5.7) give, provided $r$ is large enough,

$$
\omega\left(z_{j}, \lambda_{j}^{*}, D_{j}\right) \geqslant c / p_{j} \geqslant c r^{-\rho(F)-1}, \quad \lambda_{j}^{*}=\lambda_{j} \cap A(4) .
$$

By (5.8), $\lambda_{j}^{*}$ is mapped by $v_{j}$ into a finite union of sub-arcs of the unit circle of total length at least $c / p_{j}$ and so is mapped by $F$ into a union of sub-arcs of $J_{0}$ of total length at least $c$, using $(5.1)$ again. Let $\phi_{j}(t)$ be the angular measure of the intersection of $E_{j}$ with the circle $S(0, t)$. The above reasoning gives at least $N$ of the $E_{j}$, say $E_{1}, \ldots, E_{N}$, each having

$$
\int_{2 r^{4}}^{(1 / 2) r^{8}} \frac{\mathrm{d} t}{t \phi_{j}(t)}>c N \log r, \quad \int_{2 r^{1 / 8}}^{(1 / 2) r^{1 / 4}} \frac{\mathrm{d} t}{t \phi_{j}(t)}>c N \log r .
$$

We know that $V$ maps $E_{j}$ univalently onto $\Delta$, with $\lambda_{j}^{*}$ mapped onto a union $\mu_{j}$ of sub-arcs of the unit circle of total length at least $c$. Hence

$$
\omega\left(w, \mu_{j}, \Delta\right) \geqslant c(1-|w|)
$$


for $|w|<1$. If $z$ lies in $E_{j} \backslash A(8)$, then, because $\lambda_{j}^{*}$ lies in $A(4)$, Lemma 2.3 and (5.9) imply that

$$
\begin{aligned}
\omega\left(V(z), \mu_{j}, \Delta\right) & =\omega\left(z, \lambda_{j}^{*}, E_{j}\right) \\
& \leqslant c \exp \left(-\pi \int_{2 r^{4}}^{(1 / 2) r^{8}} \frac{\mathrm{d} t}{t \phi_{j}(t)}\right)+c \exp \left(-\pi \int_{2 r^{1 / 8}}^{(1 / 2) r^{1 / 4}} \frac{\mathrm{d} t}{t \phi_{j}(t)}\right) \\
& \leqslant c \exp (-c N \log r) .
\end{aligned}
$$

(5.3) now follows using (5.10).

\section{Proof of Theorem 1.4}

We assume that $f$ is meromorphic of finite order $\rho(f)$, and that $f$ has infinitely many poles, while $f^{\prime \prime}$ has finitely many zeros. We apply the reasoning of $\S 5$, with $F=f^{\prime}$, and retain the notation there. Let the finite asymptotic values of $f^{\prime}$ be $a_{n}$, repeated according to how often they occur as direct transcendental singularities of $\left(f^{\prime}\right)^{-1}$. Choose a path $\Gamma$, starting at 0 and tending to infinity, such that $f^{\prime}(z) \rightarrow a_{n_{0}}$ as $z$ tends to infinity on $\Gamma$. Next choose $d_{1}, d_{2}$ with $0<d_{2} \leqslant d_{1}$ such that:

(i) for each $n$, there are no asymptotic values of $f^{\prime}$ in $0<\left|w-a_{n}\right| \leqslant d_{1}$; and

(ii) for each $n$, there are no critical values of $f^{\prime}$ in $0<\left|w-a_{n}\right| \leqslant d_{2}$.

Obviously, $d_{1}$ depends only on the $a_{n}$, while $d_{2}$ depends also on $f$.

For each $n$, define a domain $V_{n}$ as follows. From the annulus $A\left(a_{n}, 0, d_{1}\right)$ delete, for each finite critical value $b_{m} \neq a_{n}$ of $f^{\prime}$, the half-open line segment

$$
w=a_{n}+s \mathrm{e}^{\mathrm{i} \arg \left(b_{m}-a_{n}\right)}, \quad d_{2} \leqslant s<d_{1} .
$$

The following lemma is an immediate consequence of Lemma 4.1 and the discussion preceding it.

Lemma 6.1. Choose $\varepsilon_{0}>0$ such that $\left|a_{n}-a_{m}\right|>4 \varepsilon_{0}$ for $a_{n} \neq a_{m}$. There exist a positive constant $\varepsilon_{1}$ and, for each $n$, an unbounded simply connected domain $U_{n}$, a component of the set $\left(f^{\prime}\right)^{-1}\left(V_{n}\right)$, such that $U_{n}$ contains a path tending to infinity on which $f^{\prime}(z)$ tends to $a_{n}$. Further, $f^{\prime}(z) \neq a_{n}$ on $U_{n}$ and $\left|f(z)-a_{n} z\right|<\varepsilon_{0}|z|$ for all large $z$ in $U_{n}$ with $\left|f^{\prime}(z)-a_{n}\right|<\varepsilon_{1}$. The constant $\varepsilon_{1}$ depends only on the asymptotic values of $f^{\prime}$.

Now let $\varepsilon_{2}$ be such that, for each $n$, if $\left|h_{1}(w)-h_{1}\left(a_{n}\right)\right| \leqslant \varepsilon_{2}$, then $\left|w-a_{n}\right|<\varepsilon_{1}$, in which $\varepsilon_{1}$ is as determined in Lemma 6.1. Next, let $\varepsilon_{3}$ be positive but so small that $\left|w-a_{n}\right|<\varepsilon_{3}$ implies that $\left|h_{1}(w)-h_{1}\left(a_{n}\right)\right|<\frac{1}{4} \varepsilon_{2}$, for $n=1,2$. Both $\varepsilon_{2}$ and $\varepsilon_{3}$ depend only on the $a_{n}$. Let $p, q$ be such that $a_{p} \neq a_{q}$ and, for $n=p, q$, let $W_{n}$ be a component of the set $\left\{z \in U_{n}:\left|f^{\prime}(z)-a_{n}\right|<\varepsilon_{3}\right\}$. For $r \geqslant r_{0}$, with $r_{0}$ large, let $\psi(r)$ be the angular measure of the intersection of $S(0, r)$ with the complement of $W_{p} \cup W_{q}$. 
Lemma 6.2. There exists a positive constant $C$, depending only on the asymptotic values of $f^{\prime}$, such that for large $r$ the number of distinct poles of $f$ in the annulus $A(2)$, as defined by (5.2), is at most

$$
C+C \int_{r_{0}}^{r^{8}} \frac{\psi(t)}{t} \mathrm{~d} t .
$$

Proof. Suppose that $r$ is large and that $A(2)$ contains $N_{1}$ distinct poles of $f$, where

$$
1+\int_{r_{0}}^{r^{8}} \frac{\psi(t)}{t} \mathrm{~d} t=o\left(N_{1}\right) .
$$

Applying Lemma 5.1 we obtain $N \geqslant c_{0} N_{1}$ distinct type II components $E_{j}$ of the set $\left(f^{\prime}\right)^{-1}\left(B_{1}\right)$, each satisfying (5.3). Since there are finitely many $a_{n}$, we may assume that $a_{1} \neq a_{2}$ and that $a_{1}, a_{2}$ are each asymptotic values of $f^{\prime}$ in each $E_{j}$. For $n=1,2$, as $w$ tends to $a_{n}$ along a path in $B_{1}$, the pre-image in $E_{j}$ tends to infinity in $U_{n}$. Provided $r$ is large enough, (5.3) shows that $A(8)$ contains the pre-image $H_{j}$ under $V=h_{1} \circ f^{\prime}$ of the $\operatorname{disc} B\left(0,1-\frac{1}{2} \varepsilon_{2}\right)$, for $1 \leqslant j \leqslant N$.

We may also assume that $r$ is so large that none of the $H_{j}$ meet the path $\Gamma$ chosen prior to Lemma 6.1, on which $f^{\prime}(z) \rightarrow a_{n_{0}}$ as $z \rightarrow \infty$. Defining an analytic and univalent branch of $\zeta=\log z$ on the complement of the path $\Gamma$, the regions $\zeta\left(H_{j}\right)$ are disjoint and, since the $H_{j}$ all lie in the intersection of $A(8)$ with the complement of $W_{p} \cup W_{q},(6.1)$ shows that at least one of the $\zeta\left(H_{j}\right)$, say $\zeta\left(H_{1}\right)$, has area $o(1)$. Using Lemma 2.1 , the pre-image in $\zeta\left(H_{1}\right)$ under $V \circ \exp$ of the line segment $w=t h_{1}\left(a_{n}\right), 0 \leqslant t \leqslant 1-\frac{3}{4} \varepsilon_{2}$, has length $o(1)$. This allows us to choose a path $\gamma^{*}$ in $\zeta\left(H_{1}\right)$, of length $o(1)$, such that the path $\gamma=\exp \left(\gamma^{*}\right)$ in $H_{1}$ joins $\eta_{1}$ to $\eta_{2}$, and such that

$$
\left|V\left(\eta_{n}\right)-h_{1}\left(a_{n}\right)\right| \leqslant \frac{3}{4} \varepsilon_{2}, \quad n=1,2 .
$$

By the choice of $\varepsilon_{2}$, there are points $\eta_{n}^{*}$ arbitrarily close to $\eta_{n}$, with $f^{\prime}\left(\eta_{n}^{*}\right) \in V_{n}$. By the choice of $J$ and $V_{n}$, there exists a path $\sigma_{0}$ in $V_{n} \cap B_{1}$ which starts at $f^{\prime}\left(\eta_{n}^{*}\right)$ and tends to $a_{n}$. Thus $\eta_{n}^{*} \in U_{n}$, and Lemma 6.1 gives

$$
\left|f\left(\eta_{n}\right)-a_{n} \eta_{n}\right| \leqslant \varepsilon_{0}\left|\eta_{n}\right|, \quad n=1,2 .
$$

We estimate the length of $\gamma$. Since $\gamma^{*}$ has length $o(1)$, we have $z=(1+o(1)) \eta_{1}$ for all $z$ on $\gamma$ and

$$
\int_{\gamma}|\mathrm{d} z|=\int_{\gamma^{*}}|z||\mathrm{d} \zeta|=o\left(\left|\eta_{1}\right|\right)
$$

But $f^{\prime}$ maps $\gamma$ into the bounded domain $B_{1}$, and so

$$
f\left(\eta_{2}\right)-f\left(\eta_{1}\right)=\int_{\gamma} f^{\prime}(z) \mathrm{d} z=o\left(\left|\eta_{1}\right|\right) .
$$

Since $a_{1} \neq a_{2}$, this contradicts (6.2), and Lemma 6.2 is proved. 
We now complete the proof of Theorem 1.4. By Lemma 6.2 and the fact that $\psi(t) \leqslant 2 \pi$, there exist positive $C_{j}$ depending only on the $a_{n}$ such that, for all large $r$,

$$
\bar{n}\left(r^{2}, f\right)-\bar{n}\left(r^{1 / 2}, f\right) \leqslant C_{1}+C_{1} \int_{r_{0}}^{r^{8}} \frac{\psi(t)}{t} \mathrm{~d} t \leqslant C_{2} \log r \leqslant C_{3}\left(\log r^{2}-\log r^{1 / 2}\right) .
$$

Thus $\bar{n}(r, f) \leqslant C_{4} \log r$, and Theorem 1.4 is proved.

\section{Proof of Theorem 1.5}

We assume that $f$ is meromorphic in the plane of order less than $1+\varepsilon$, where $0<\varepsilon<\frac{1}{2}$, and that $f^{\prime \prime}$ has finitely many zeros but $f$ has infinitely many poles. We retain the notation of the previous section. By the discussion in $\S 5, f^{\prime}$ has at least two distinct finite asymptotic values $a_{1}, a_{2}$. By the Denjoy-Carleman-Ahlfors Theorem $[\mathbf{3}, \mathbf{2 5}]$, these are the only asymptotic values of $f^{\prime}$. Hence we may assume that $a_{p}=a_{1}=1, a_{q}=a_{2}=-1$.

Lemma 7.1. We have

$$
\int_{r_{0}}^{r} \frac{\psi(t)}{t} \mathrm{~d} t \leqslant 2 \pi \varepsilon \log r, \quad \bar{n}(r, f) \leqslant C \varepsilon \log r, \quad r \rightarrow \infty,
$$

in which $C$ is a positive absolute constant, in particular not depending on $\varepsilon$.

Proof. For $n=1,2$, define the following. For $r \geqslant r_{0}$, let $\psi_{n}(r)$ be the angular measure of the intersection of $W_{n}$ with the circle $|z|=r$. Let $u_{n}(z)$ be defined by $u_{n}(z)=$ $\log \left|\varepsilon_{3} /\left(f^{\prime}(z)-a_{n}\right)\right|$ for $z$ in $W_{n}$, with $u_{n}(z)=0$ for $z$ outside $W_{n}$. Then $u_{1}$ and $u_{2}$ are subharmonic in the plane and Lemma 2.3 gives

$$
\int_{r_{0}}^{r} \frac{\pi}{t \psi_{n}(t)} \mathrm{d} t \leqslant \log B_{0}\left(2 r, u_{n}\right)+O(1) \leqslant(1+\varepsilon) \log r
$$

as $r \rightarrow \infty$, for $n=1,2$. But, for $t \geqslant r_{0}$,

$$
\frac{\pi}{\psi_{1}(t)}+\frac{\pi}{\psi_{2}(t)} \geqslant \frac{4 \pi}{\psi_{1}(t)+\psi_{2}(t)}=\frac{4 \pi}{2 \pi-\psi(t)} \geqslant 2+\frac{\psi(t)}{\pi} .
$$

This proves the first assertion of Lemma 7.1, and the second follows as in the previous section. The following is a simple consequence of Lemma 2.3 .

Lemma 7.2. There exists $L_{0}>1$ such that the following is true. Let $r>0, L>1$ and let $\gamma_{r}$ be a simple piecewise smooth path which, apart from its endpoints, lies in $r<|z|<L r$ and which joins $|z|=r$ to $|z|=L r$. Let $U_{r}=\left\{z: r<|z|<L r, z \notin \gamma_{r}\right\}$. Then if $L \geqslant L_{0}$ we have

$$
\omega\left(z, S(0, r), U_{r}\right)+\omega\left(z, S(0, L r), U_{r}\right)<\frac{1}{2}, \quad z \in U_{r}, \quad|z|=L^{1 / 2} r .
$$


Let $L \geqslant L_{0}$, with $L_{0}$ as in Lemma 7.2. By (2.3) and (7.1), the sets

$$
K_{1}=\left\{r \geqslant r_{0}: \psi(r) \geqslant \varepsilon^{1 / 2}\right\}, \quad K_{2}=\left\{r \geqslant r_{0}: \psi(L r) \geqslant \varepsilon^{1 / 2}\right\}
$$

each have upper logarithmic density at most $2 \pi \varepsilon^{1 / 2}$. Next we note that by Lemma 2.5 the set

$$
K_{3}=\left\{r \geqslant 1: T\left(L^{2} r, f^{\prime \prime}\right) \geqslant L^{6} T\left(r, f^{\prime \prime}\right)\right\}
$$

has upper logarithmic density at most $\frac{2}{3}$. Further, (7.1) gives

$$
h(r)=\exp (\bar{n}(r, f))=O\left(r^{C \varepsilon}\right), \quad r \rightarrow \infty,
$$

and so by Lemma 2.5 again and (2.3) the set

$$
K_{4}=\left\{r \geqslant 1: \bar{n}\left(L^{2} r, f\right)>\bar{n}(r / L, f)\right\}=\left\{r \geqslant 1: h\left(L^{2} r\right) \geqslant e h(r / L)\right\}
$$

has upper logarithmic density at most $3 C \varepsilon \log L$.

Provided $\varepsilon$ is small enough we may choose arbitrarily large $r$, not in any of the exceptional sets $K_{1}, K_{2}, K_{3}, K_{4}$, and such that

$$
\left|\frac{f^{\prime \prime}(z)}{f^{\prime}(z)-a_{1}}\right|+\left|\frac{f^{\prime \prime}(z)}{f^{\prime}(z)-a_{2}}\right| \leqslant r^{c_{0}}, \quad|z|=r, L r
$$

denoting by $c_{j}$ positive constants which do not depend on $\varepsilon$. By (7.4), $f$ has no poles in $r / L<|z|<L^{2} r$. Hence, by (7.3) and a standard application of the Poisson-Jensen formula we have

$$
\log \left|f^{\prime \prime}(z)\right|<c_{1} T\left(r, f^{\prime \prime}\right), \quad r \leqslant|z| \leqslant L r,
$$

since $L$ does not depend on $\varepsilon$. Further, by (7.2) and (7.5), we have

$$
\log \left|f^{\prime \prime}(z)\right|<c_{2} \log r, \quad z \in\left(S(0, r) \backslash T_{r}\right) \cup\left(S(0, L r) \backslash T_{L r}\right),
$$

in which $T_{r} \subseteq S(0, r)$ and $T_{L r} \subseteq S(0, L r)$, each having angular measure at most $\varepsilon^{1 / 2}$.

Choose a simple piecewise smooth curve $\gamma_{r}$ on which

$$
\log \left|f^{\prime \prime}(z)\right|<-\left(\frac{1}{2}\right) T\left(r, f^{\prime \prime}\right)
$$

such that $\gamma_{r}$ joins $|z|=r$ to $|z|=L r$ and, apart from its endpoints, lies in $r<|z|<L r$. Such a curve exists by the maximum principle applied to $1 / f^{\prime \prime}$. Define $U_{r}$ as in Lemma 7.2, so that

$$
\omega\left(z, \gamma_{r}, U_{r}\right)>\frac{1}{2}, \quad z \in U_{r}, \quad|z|=L^{1 / 2} r .
$$

For $z$ in $U_{r}$ with $|z|=L^{1 / 2} r$,

$$
\omega\left(z, T_{L r}, U_{r}\right) \leqslant \omega\left(z, T_{L r}, B(0, L r)\right)<c_{3} \varepsilon^{1 / 2},
$$

and the change of variables $\zeta=1 / z$ shows that the same estimate holds for $\omega\left(z, T_{r}, U_{r}\right)$. Hence, (7.6), (7.7), (7.8) and (7.9) give

$$
\log \left|f^{\prime \prime}(z)\right|<\left(-\frac{1}{4}+c_{4} \varepsilon^{1 / 2}\right) T\left(r, f^{\prime \prime}\right),
$$

so that $f^{\prime \prime}(z)$ is small on the whole circle $|z|=L^{1 / 2} r$, provided $\varepsilon$ is small enough. This contradicts the existence of the distinct asymptotic values \pm 1 of $f^{\prime}$ and Theorem 1.5 is proved. 


\section{Proof of Theorem 1.6}

Assume that $f$ satisfies the hypotheses of Theorem 1.6, but has infinitely many poles. By Theorem 1.4 we have (1.2). Let the finite asymptotic values of $f^{(k-1)}$ be $a_{n}$, repeated according to how often they occur as direct transcendental singularities of the inverse function of $f^{(k-1)}$. By $\S 5$ there are at least two distinct $a_{n}$. If the positive constant $\varepsilon_{0}$ is small enough, then to each $a_{n}$ corresponds, as in $\S 4$, an unbounded simply connected component $U_{n}$ of the set $\left\{z:\left|f^{(k-1)}(z)-a_{n}\right|<\varepsilon_{0}\right\}$, lying in $\{z:|z|>2\}$, such that $f^{(k-1)}(z) \neq a_{n}$ on $U_{n}$ and

$$
\left|f^{(k-2)}(z)-a_{n} z\right| \leqslant C_{1}|z|\left|f^{(k-1)}(z)-a_{n}\right|+O(1), \quad z \in U_{n},
$$

in which $C_{1}$ is a positive constant not depending on $a_{n}$ or $f$.

Lemma 8.1. Choose a large positive integer $N$ and for each $n$ let the subharmonic function $u_{n}$ be defined as in Lemma 4.1 by $u_{n}(z)=\log ^{+}\left|d_{n} /\left(z^{N}\left(f^{(k-1)}(z)-a_{n}\right)\right)\right|$ for $z$ in $U_{n}$, with $u_{n}(z)=0$ otherwise, and with $d_{n}$ a small positive constant.

Then $U_{n}$ contains finitely many components $W_{j, n}$ of the set $\left\{z: u_{n}(z)>0\right\}$, each simply connected, and we have

$$
f^{(\nu)}(z)=O\left(|z|^{k-\nu-1}\right), \quad z \in W_{j, n}, \quad \nu=0, \ldots, k-2 .
$$

Each $u_{n}$ has lower order at least $1 /(2-1 / \rho)$.

Proof. The estimate (8.2) will be proved by applying (4.1) to $f^{(k-2)}(z)-a_{n} z$. Fixing $z^{*}$ in $W_{j, n}$, choose a polynomial $P_{n}$ of degree at most $k-1$ such that

$$
f^{(\nu)}(z)=P_{n}^{(\nu)}(z)+\int_{z^{*}}^{z} \frac{(z-t)^{k-\nu-2}}{(k-\nu-2) !}\left(f^{(k-1)}(t)-a_{n}\right) \mathrm{d} t, \quad z \in W_{j, n}, \quad 0 \leqslant \nu \leqslant k-2 .
$$

Expanding out the $(z-t)^{k-\nu-2}$ term in (8.3), and using (4.1), we obtain (8.2).

To prove that each $u_{n}$ has lower order at least $1 /(2-1 / \rho)$, assume without loss of generality that $a_{1} \neq a_{2}$ and, for $n=1,2$ and $t>0$, let $\theta_{n}^{*}(t)$ be the angular measure of the intersection of $U_{n}$ with the circle $S(0, t)$. Proceeding as in [28, Lemma 3], the Cauchy-Schwarz inequality gives

$$
\left(\int_{1}^{r} \frac{\pi}{t \theta_{n}^{*}(t)} \mathrm{d} t\right)\left(\int_{1}^{r} \frac{\theta_{n}^{*}(t)}{t \pi} \mathrm{d} t\right) \geqslant(\log r)^{2}, \quad r \rightarrow \infty, \quad n=1,2 .
$$

But, by Lemma 2.3, for large $r$,

$$
(\rho+o(1)) \log r \geqslant \log B_{0}\left(2 r, u_{2}\right)+O(1) \geqslant \int_{1}^{r} \frac{\pi}{t \theta_{2}^{*}(t)} \mathrm{d} t .
$$

Thus

$$
\int_{1}^{r} \frac{\theta_{2}^{*}(t)}{t \pi} \mathrm{d} t \geqslant(\rho+o(1))^{-1} \log r, \quad \int_{1}^{r} \frac{\theta_{1}^{*}(t)}{t \pi} \mathrm{d} t \leqslant\left(2-(\rho+o(1))^{-1}\right) \log r
$$


so that

$$
\log B_{0}\left(2 r, u_{1}\right)+O(1) \geqslant \int_{1}^{r} \frac{\pi}{t \theta_{1}^{*}(t)} \mathrm{d} t \geqslant\left(\frac{1}{2-1 / \rho}-o(1)\right) \log r
$$

Lemma 8.2. Choose $\rho_{j}$ with $\rho_{0}<\rho_{1}<\cdots<\rho_{9}<1 /(2-1 / \rho)$, and let $\delta_{1}$ be a small positive constant. Then there exists $\delta_{2}>0$ such that the following is true. If $H_{0}$ is a subset of $[1, \infty)$ of finite measure, then for each sufficiently large $r$ and each $n$ there exists $s \notin H_{0}$ such that

$$
r^{1+\delta_{1}} \leqslant s \leqslant r^{1+2 \delta_{1}}, \quad u_{n}(z)>r^{\rho_{8}}, \quad z \in H_{n}(r),
$$

in which $H_{n}(r)$ is a subset of the circle $|z|=s$, of angular measure at least $\delta_{2}$.

Proof. Using Lemma 8.1, take $\zeta_{0}$ with $\left|\zeta_{0}\right|=r$ and $u_{n}\left(\zeta_{0}\right)>r^{\rho_{9}}$, and let $D_{0}$ be the component of the set $\left\{z \in U_{n}: u_{n}(z)>r^{\rho 8}\right\}$ in which $\zeta_{0}$ lies. Let $\theta(t)$ be the angular measure of the intersection of $D_{0}$ with the circle $|z|=t$. Since $u_{n}$ has order at most $\rho$, Lemma 2.3 gives

$$
r^{\rho_{9}} \leqslant u_{n}\left(\zeta_{0}\right) \leqslant r^{\rho_{8}}+r^{(1+\rho)\left(1+2 \delta_{1}\right)} \exp \left(-\pi \int_{2 r^{1+\delta_{1}}}^{(1 / 2) r^{1+2 \delta_{1}}} \frac{\mathrm{d} t}{t \theta(t)}\right)
$$

and Lemma 8.2 follows.

Lemma 8.3. Let $a_{n} \neq 0$ and let $K$ be a large positive constant. Let the positive function $\eta(r)$ tend to 0 slowly as $r \rightarrow \infty$. Then for all sufficiently large $r$, at least one of the sets

$$
\begin{aligned}
& \Omega_{r}^{+}=\{z: r / K \leqslant|z| \leqslant K r, \eta(r) \leqslant \arg z \leqslant \pi-\eta(r)\}, \\
& \Omega_{r}^{-}=\{z: r / K \leqslant|z| \leqslant K r, \pi+\eta(r) \leqslant \arg z \leqslant 2 \pi-\eta(r)\}
\end{aligned}
$$

is a subset of one of the $W_{j, n}$.

Proof. Using (1.2) and (1.3), write

$$
\frac{f^{(k)}(z)}{f^{(m)}(z)}=\frac{h_{1}(z)}{h_{2}(z)}
$$

in which $h_{1}$ is analytic outside the region $\left|\arg z^{2}\right| \leqslant \phi(|z|)$, and $h_{2}$ is entire of order less than $\rho_{0}$. Choose a family of discs $B_{\nu}$, with finite sum of radii, and a positive constant $M_{1}$, such that for all $z$ not in the union $H^{*}$ of the $B_{\nu}$ we have

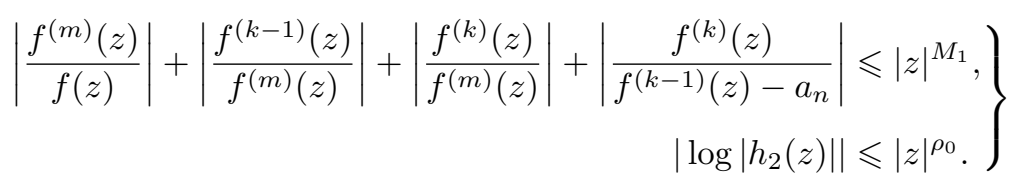


Further, choose a small positive $\delta_{1}$ and $s=s_{n}$ satisfying (8.4). We may assume without loss of generality that the part $H_{n}^{*}(r)$ of $H_{n}(r)$ lying in $\{z: \eta(r) \leqslant \arg z \leqslant \pi-\eta(r)\}$ has angular measure at least $\delta_{3} \geqslant \delta_{2} / 4$, in which $\delta_{2}$ is as in Lemma 8.2. We may choose $s$, as well as $r_{1}$ and $\lambda$ with

$$
K^{-6} r \leqslant r_{1} \leqslant K^{-4} r, \quad \eta(r) / 8<\lambda<\eta(r) / 4,
$$

such that

$$
\partial \Omega(s, 4 s, \lambda) \cap H^{*}=\partial \Omega\left(r_{1}, 2 s, 2 \lambda\right) \cap H^{*}=\partial \Omega\left(K^{2} r_{1}, s, 4 \lambda\right) \cap H^{*}=\emptyset,
$$

in which

$$
\Omega\left(t_{1}, t_{2}, t_{3}\right)=\left\{z: t_{1}<|z|<t_{2}, t_{3}<\arg z<\pi-t_{3}\right\} .
$$

Since $\delta_{1}$ is small, we have, by (8.5) and (8.6),

$$
\log \left|h_{1}(z)\right| \leqslant r^{\rho_{1}}, \quad z \in \partial \Omega(s, 4 s, \lambda) \cup \partial \Omega\left(r_{1}, 2 s, 2 \lambda\right) .
$$

We apply the two-constants theorem to $\log \left|h_{1}(z)\right|$ on the region $\Omega(s, 4 s, \lambda)$. Since

$$
\frac{f^{(k)}}{f^{(m)}}=\left(\frac{f^{(k)}}{f^{(k-1)}-a_{n}}\right)\left(\frac{f^{(k-1)}-a_{n}}{f^{(k-1)}}\right)\left(\frac{f^{(k-1)}}{f^{(m)}}\right)
$$

(8.4) and (8.6) give

$$
\log \left|h_{1}(z)\right|<-r^{\rho_{7}}, \quad z \in H_{n}^{*}(r) .
$$

Thus, (8.7) and a standard estimate for harmonic measure lead to

$$
\log \left|h_{1}(z)\right|<-r^{\rho_{6}}, \quad z \in E_{0}=\{z:|z|=2 s, \pi / 8 \leqslant \arg z \leqslant 7 \pi / 8\} .
$$

By Lemma 2.4 and a simple change of variables,

$$
\omega\left(z, E_{0}, \Omega\left(r_{1}, 2 s, 2 \lambda\right)\right) \geqslant r^{-6 \delta_{1}}, \quad z \in \partial \Omega\left(K^{2} r_{1}, s, 4 \lambda\right) .
$$

Hence, using (8.6), (8.7) and (8.8) we have

$$
\log \left|h_{1}(z)\right|<-r^{\rho_{5}}, \quad\left|\frac{f^{(k)}(z)}{f^{(m)}(z)}\right| \leqslant \exp \left(-r^{\rho_{4}}\right), \quad z \in \partial \Omega\left(K^{2} r_{1}, s, 4 \lambda\right) .
$$

We estimate $f$ on $\partial \Omega\left(K^{2} r_{1}, s, 4 \lambda\right)$. Choose $z_{1}$ in $H_{n}^{*}(r)$ and so in $\partial \Omega\left(K^{2} r_{1}, s, 4 \lambda\right) \cap W_{j, n}$, and a polynomial $P_{1}$ such that $P_{1}^{(\nu)}\left(z_{1}\right)=f^{(\nu)}\left(z_{1}\right)$ for $0 \leqslant \nu \leqslant k-1$. Then we may write

$$
f(z)=P_{1}(z)+\int_{z_{1}}^{z} \frac{(z-t)^{k-1}}{(k-1) !} f^{(k)}(t) \mathrm{d} t=P_{1}(z)+\int_{z_{1}}^{z} \eta(t) f(t) \mathrm{d} t
$$

in which, using (8.2), (8.6) and (8.9), for some $M_{2}>0$ independent of $r$ and $K$,

$$
\left|P_{1}(z)\right| \leqslant r^{M_{2}}, \quad|\eta(t)| \leqslant \exp \left(-r^{\rho_{3}}\right), \quad z \in \partial \Omega\left(K^{2} r_{1}, s, 4 \lambda\right) .
$$


A standard application of Gronwall's Lemma [14] and (8.6) and (8.9) give

$$
\left.\begin{array}{rl}
\log ^{+}|f(z)| & =O(\log r), \\
\log ^{+}\left|f^{(m)}(z)\right| & =O(\log r),
\end{array}\right\} \quad\left|f^{(k)}(z)\right| \leqslant \exp \left(-r^{\rho_{2}}\right), \quad \text { for } z \in \partial \Omega\left(K^{2} r_{1}, s, 4 \lambda\right) .
$$

Since $z_{1}$ is in $H_{n}(r)$, a further integration shows that $\partial \Omega\left(K^{2} r_{1}, s, 4 \lambda\right)$ is a subset of $W_{j, n}$, and so is $\Omega\left(K^{2} r_{1}, s, 4 \lambda\right)$, since $W_{j, n}$ is simply connected. This proves Lemma 8.3.

Lemma 8.4. We have $\bar{N}(r, f) \neq o(\log r)^{2}$ as $r \rightarrow \infty$.

Proof. Suppose on the contrary that $\bar{N}(r, f)=o(\log r)^{2}$ as $r \rightarrow \infty$. Then

$$
T\left(r, f^{(k+1)} / f^{(k)}\right) \leqslant \bar{N}(r, f)+O(\log r)=o(\log r)^{2} .
$$

It follows from Lemma 2 of [20] that there exist sequences $R_{\mu} \rightarrow \infty$ and $S_{\mu} \rightarrow \infty$ such that

$$
\frac{f^{(k+1)}(z)}{f^{(k)}(z)}=\beta_{\mu} z^{\tau_{\mu}}(1+o(1)), \quad S_{\mu}^{-2} R_{\mu} \leqslant|z| \leqslant S_{\mu}^{2} R_{\mu}
$$

in which each $\tau_{\mu}$ is an integer and each $\beta_{\mu}$ is a non-zero complex number. There is no loss of generality in assuming that both $R_{\mu}$ and $2 R_{\mu}$ are normal for the Wiman-Valiron theory $[\mathbf{1 1}, \mathbf{3 1}]$ applied to $1 / f^{(k)}$, for otherwise we may adjust $R_{\mu}$ and make $S_{\mu}$ slightly smaller. Since the central index $\sigma(r)$ of $1 / f^{(k)}$ is non-decreasing, (2.4) gives $\tau_{\mu} \geqslant-1$ for each $\mu$. We may also assume that

$$
\frac{f^{(k)}(z)}{f^{(k-1)}(z)-a_{n}}=O\left(R_{\mu}^{M}\right), \quad|z|=R_{\mu}
$$

for all finite asymptotic values $a_{n}$ of $f^{(k-1)}$ and for some fixed $M$, independent of $\mu$.

Case 1. Suppose that $\tau_{\mu}=-1$.

In this case, (2.4) shows that we may assume without loss of generality that $\beta_{\mu}=$ $-N_{1}=-\sigma\left(R_{\mu}\right)$. Integration of (8.10) gives, with $C$ a non-zero constant,

$$
1 / f^{(k)}(z)=C\left(z / R_{\mu}\right)^{N_{1}} \mathrm{e}^{o\left(N_{1}\right)}, \quad 2 R_{\mu} \leqslant|z| \leqslant 3 R_{\mu} .
$$

Since $M\left(2 R_{\mu}, 1 / f^{(k)}\right)$ is large, this implies that $C\left(\frac{5}{2}\right)^{N_{1}}$ must be large. Thus $f^{(k)}(z)=$ $O\left(R_{\mu}^{-2}\right)$ on $|z|=3 R_{\mu}$ and a further integration leads to a contradiction to the established fact that $f^{(k-1)}$ has at least two asymptotic values.

Case 2. Suppose that $\tau_{\mu} \geqslant 0$.

Choose $z_{1}, z_{2}$ with

$$
\left|z_{1}\right|=R_{\mu}, \quad\left|z_{2}\right|=R_{\mu} S_{\mu}^{-1}, \quad\left|1 / f^{(k)}\left(z_{j}\right)\right|=M\left(\left|z_{j}\right|, 1 / f^{(k)}\right) .
$$

Next, choose a branch of $\log f^{(k)}(z)$ with

$$
\left|\operatorname{Im}\left(\log f^{(k)}\left(z_{2}\right)\right)\right| \leqslant \pi
$$


For $z$ with

$$
R_{\mu} S_{\mu}^{-1} \leqslant|z| \leqslant R_{\mu} S_{\mu}, \quad-\pi<\arg \left(z / z_{1}\right) \leqslant \pi
$$

we integrate by parts along the straight line from $z_{2}$ to $z_{2}\left|z / z_{2}\right|$ and then around an arc of the circle $|\zeta|=|z|$ to obtain

$$
\begin{aligned}
\log f^{(k)}(z)=\log f^{(k)}\left(z_{2}\right)+\frac{\beta_{\mu} z^{\tau_{\mu}+1}}{\tau_{\mu}+1} & (1+o(1)) \\
& -\frac{\beta_{\mu} z_{2}^{\tau_{\mu}+1}}{\tau_{\mu}+1}(1+o(1))+\int_{z_{2}}^{z} \frac{\beta_{\mu} \zeta^{\tau_{\mu}+1}}{\tau_{\mu}+1} o\left(\left|\zeta^{-1}\right|\right) \mathrm{d} \zeta .
\end{aligned}
$$

Thus, for $z$ satisfying (8.13),

$$
\log f^{(k)}(z)=D+A z^{N}(1+o(1))
$$

in which

$$
D=\log f^{(k)}\left(z_{2}\right)-\frac{\beta_{\mu} z_{2}^{\tau_{\mu}+1}}{\tau_{\mu}+1}, \quad A=\frac{\beta_{\mu}}{\tau_{\mu}+1}, \quad N=\tau_{\mu}+1 .
$$

We set $T_{\mu}=R_{\mu} S_{\mu}^{-3 / 4}$ and distinguish two subcases.

Case 2(a). Suppose that $\left|A T_{\mu}^{N}\right|<\left|\log f^{(k)}\left(z_{2}\right)\right|$.

Then using (8.12) and the fact that $\tau_{\mu} \geqslant 0$,

$\log f^{(k)}(z)=D(1+o(1))=(1+o(1)) \log \left|f^{(k)}\left(z_{2}\right)\right|, \quad|z|=R_{\mu} S_{\mu}^{-1}, \quad-\pi<\arg \left(z / z_{1}\right) \leqslant \pi$, and $f^{(k)}(z)=O\left(|z|^{-2}\right)$ on $|z|=R_{\mu} S_{\mu}^{-1}$, a contradiction arising as in Case 1 .

Case 2(b). Suppose that $\left|A T_{\mu}^{N}\right| \geqslant\left|\log f^{(k)}\left(z_{2}\right)\right|$.

Then $\left|A T_{\mu}^{N}\right|$ is large and (8.14) becomes

$$
\log f^{(k)}(z)=A z^{N}(1+o(1)), \quad R_{\mu} S_{\mu}^{-1 / 2} \leqslant|z| \leqslant R_{\mu} S_{\mu}^{1 / 2}, \quad-\pi<\arg \left(z / z_{1}\right) \leqslant \pi .
$$

But $f^{(k)}(z)$ is small on an arc of $|z|=R_{\mu}$ of angular measure at least $\pi-o(1)$, by Lemma 8.3 and (8.11), so that (8.16) gives $N=1$. However,

$$
-(1+o(1)) \frac{\sigma\left(R_{\mu}\right)}{z_{1}}=\frac{f^{(k+1)}\left(z_{1}\right)}{f^{(k)}\left(z_{1}\right)}=A(1+o(1)),
$$

by (8.10) and (8.15), since $R_{\mu}$ is normal for the Wiman-Valiron theory applied to $1 / f^{(k)}$. Thus

$$
\arg A z_{1}=\pi+o(1)
$$

Writing (8.16) in the form

$$
u=-\log f^{(k)}(z)=-A z_{1}\left(z / z_{1}\right)(1+o(1)),
$$


it follows that $u$ is univalent with

$$
\frac{\mathrm{d} u}{\mathrm{~d} z}=-A(1+o(1))
$$

on

$$
\Omega=\left\{z: R_{\mu} / 16 \leqslant|z| \leqslant 16 R_{\mu},\left|\arg \left(z / z_{1}\right)\right| \leqslant 5 \pi / 8\right\},
$$

and $u(\Omega)$ contains the region

$$
\Omega_{1}=\left\{u:|A| R_{\mu} / 8 \leqslant|u| \leqslant 8|A| R_{\mu},|\arg u| \leqslant 9 \pi / 16\right\} .
$$

Let

$$
\Omega_{2}=\left\{z: R_{\mu} / 4 \leqslant|z| \leqslant 4 R_{\mu}, \log \left|f^{(k)}(z)\right|<-2 M \log R_{\mu}\right\} .
$$

Then (8.17) and (8.18) imply that $\Omega_{2}$ is a subset of $\Omega$, and

$$
u\left(\Omega_{2}\right) \subseteq \Omega_{3}=\left\{u:|A| R_{\mu} / 8 \leqslant|u| \leqslant 8|A| R_{\mu}, \operatorname{Re}(u)>2 M \log R_{\mu}\right\} \subseteq \Omega_{1} .
$$

Using Lemma 8.1, choose distinct asymptotic values $a_{1}, a_{2}$ of $f^{(k-1)}$, and $\psi_{1}$ and $\psi_{2}$ with

$$
\left|\psi_{n}\right|=R_{\mu}, \quad \log \left|f^{(k-1)}\left(\psi_{n}\right)-a_{n}\right|<-4 M \log R_{\mu}, \quad n=1,2 .
$$

Then $\psi_{1}, \psi_{2}$ lie in $\Omega_{2}$ by (8.11), and their images under $u$ lie in $\Omega_{3}$. Thus $u\left(\psi_{1}\right)$ and $u\left(\psi_{2}\right)$ may be joined by a path $\lambda$ in $\Omega_{3}$, of length $O\left(|A| R_{\mu}\right)$. Now the pre-image $\Lambda=u^{-1}(\lambda)$ joining $\psi_{1}$ to $\psi_{2}$ has length $O\left(R_{\mu}\right)$, by (8.19), and is such that

$$
\log \left|f^{(k)}(z)\right|<-2 M \log R_{\mu}, \quad z \in \Lambda .
$$

Thus $f^{(k-1)}\left(\psi_{1}\right)-f^{(k-1)}\left(\psi_{2}\right)=o(1)$, which contradicts the choice of the $\psi_{n}$. Lemma 8.4 is proved.

As in $\S 5$, choose a closed path $J$ on which each finite asymptotic value $a_{n}$ of $f^{(k-1)}$ lies. If there are just two distinct $a_{n}$, say $a_{1}, a_{2}$, then $J$ is the circle of centre $\left(a_{1}+a_{2}\right) / 2$ and diameter $\left|a_{1}-a_{2}\right|$. Let $B_{1}$ be the interior domain of $J$, and let $B_{2}, h_{1}, h_{2}$ be defined as in $\S 5$. In particular, if $J$ is a circle, then $h_{1}$ is simply a linear transformation.

Lemma 8.5. For each type II component $E_{j}$ of the set $\left\{z: f^{(k-1)}(z) \in B_{1}\right\}$, choose $\zeta_{j} \in E_{j}$ such that $h_{1}\left(f^{(k-1)}\left(\zeta_{j}\right)\right)=0$. Let $n_{0}(r)$ be the number of $\zeta_{j}$ in $|z| \leqslant r$. Then $n_{0}(r) \neq o(\log r)$ as $r \rightarrow \infty$.

This follows at once from Lemmas 5.1 and 8.4.

Choose a large positive $L$ such that for arbitrarily large $r$ there are at least 64 distinct $\zeta_{j}$ in $A(0, r / L, L r)$. Since $w=h_{1}\left(f^{(k-1)}(z)\right)$ maps $E_{j}$ univalently onto $B(0,1)$, we may choose $G_{j}$ to be the inverse function mapping $B(0,1)$ onto $E_{j}$.

Lemma 8.6. Denote by $c_{j}$ positive constants independent of $r$ and $L$. Then

$$
c_{1} r \leqslant\left|G_{j}^{\prime}(0)\right| \leqslant c_{2} r .
$$


Proof. The right-hand estimate of (8.20) follows from the Koebe one-quarter theorem, since 0 is not in $E_{j}$. To prove the left-hand estimate, suppose that $G_{j}^{\prime}(0)=o(r)$. Let $a_{1}$, $a_{2}$ be distinct finite asymptotic values of $f^{(k-1)}$ in $E_{j}$. Koebe's distortion theorem gives a path $\gamma$, of length $o(r)$, joining $z_{p} \in U_{p}$ to $z_{q} \in U_{q}$, with $a_{p} \neq a_{q}$ and

$$
\int_{\gamma} f^{(k-1)}(z) \mathrm{d} z=o(r)
$$

which contradicts (8.1) if $\varepsilon_{0}$ was chosen small enough.

Lemma 8.7. $f^{(k-1)}$ has precisely one finite non-zero asymptotic value.

Proof. Suppose that $f^{(k-1)}$ has more than one finite non-zero asymptotic value. Then Lemma 8.3 and the Koebe one-quarter theorem applied to $G_{j}$ on $B\left(0, \frac{1}{2}\right)$ give $G_{j}^{\prime}(0)=$ $o(r)$. On the other hand, $f^{(k-1)}$ has at least two finite asymptotic values, and this proves Lemma 8.7.

We may assume henceforth that the finite asymptotic values of $f^{(k-1)}$ are 0 and 1 . Thus $J$ is the circle $S\left(\frac{1}{2}, \frac{1}{2}\right)$, while $B_{1}$ is the $\operatorname{disc} B\left(\frac{1}{2}, \frac{1}{2}\right)$, and $h_{1}(w)=2\left(w-\frac{1}{2}\right)$. Set

$$
g(z)=2\left(f^{(k-1)}(z)-\frac{1}{2}\right)=h_{1}\left(f^{(k-1)}(z)\right) .
$$

Let $\theta_{j}(t)$ be the angular measure of the intersection of $E_{j}$ with the circle $S(0, t)$. Recall that $w=g(z)$ maps $E_{j}$ univalently onto $B(0,1)$, with $g\left(\zeta_{j}\right)=0$ and inverse function $z=G_{j}(w)$. Since there are 64 of the $E_{j}$, at least one of them must be such that

$$
\int_{L r}^{L^{2} r} \frac{\mathrm{d} t}{t \theta_{j}(t)} \geqslant 4 \log L, \quad \int_{r / L^{2}}^{r / L} \frac{\mathrm{d} t}{t \theta_{j}(t)} \geqslant 4 \log L
$$

Suppose that $Z \in E_{j} \backslash A\left(0, r / L^{2}, L^{2} r\right)$ and $W=g(Z)$. Then

$$
\log \left(\frac{1+|W|}{1-|W|}\right)=2 \int_{[0, W]} \frac{|\mathrm{d} w|}{1-|w|^{2}}=2 \int_{G_{j}([0, W])} \frac{|\mathrm{d} z|}{\left(1-|w|^{2}\right)\left|G_{j}^{\prime}(w)\right|}
$$

and so Koebe's one-quarter theorem and (8.21) give

$$
\log \left(\frac{1+|W|}{1-|W|}\right) \geqslant \frac{1}{2} \int_{G_{j}([0, W])} \frac{|\mathrm{d} z|}{\operatorname{dist}\left\{z, \partial E_{j}\right\}} \geqslant \int_{G_{j}([0, W])} \frac{|\mathrm{d} z|}{|z| \theta_{j}(|z|)} \geqslant 4 \log L,
$$

since $\zeta_{j}=G_{j}(0) \in A(0, r / L, L r)$. Define $v_{1}, v_{2}$ by

$$
v_{\mu}=G_{j}\left(t_{\mu}\right), \quad t_{1}=-1+L^{-3}, \quad t_{2}=1-L^{-3} .
$$

Then (8.22) gives

$$
H_{0}=G_{j}\left(\left[t_{1}, t_{2}\right]\right) \subseteq A\left(0, r / L^{2}, L^{2} r\right)
$$

Let

$$
h(z)=2 f^{(k-2)}(z)-z, \quad h^{\prime}(z)=g(z)
$$


Using Lemma 4.1, we obtain

$$
\left|h\left(v_{1}\right)+v_{1}\right| \leqslant c_{3}\left|v_{1}\right| L^{-3}, \quad\left|h\left(v_{2}\right)-v_{2}\right| \leqslant c_{3}\left|v_{2}\right| L^{-3} .
$$

Integration by parts gives

$$
h\left(v_{2}\right)-h\left(v_{1}\right)=\int_{H_{0}} g(z) \mathrm{d} z=v_{2} g\left(v_{2}\right)-v_{1} g\left(v_{1}\right)-\int_{H_{0}} z g^{\prime}(z) \mathrm{d} z .
$$

Thus, using (8.23), (8.24) and (8.25),

$$
\left|\int_{\left[t_{1}, t_{2}\right]} z \mathrm{~d} w\right|=\left|\int_{H_{0}} z g^{\prime}(z) \mathrm{d} z\right| \leqslant\left|h\left(v_{1}\right)-v_{1} g\left(v_{1}\right)\right|+\left|h\left(v_{2}\right)-v_{2} g\left(v_{2}\right)\right| \leqslant c_{4} r L^{-1} \text {. }
$$

But Lemmas 8.3 and 8.6 and the Koebe Theorems give, without loss of generality, $\operatorname{Im}\left(\zeta_{j}\right)>c_{5} r$, and $\operatorname{Im}\left(G_{j}(t)\right)>c_{6} r$ for $-c_{7} \leqslant t \leqslant c_{7}$, while $\operatorname{Im}\left(G_{j}(t)\right)>-o(r)$ for $t_{1} \leqslant t \leqslant t_{2}$. This contradicts (8.26) and Theorem 1.6 is proved.

Acknowledgements. The author acknowledges valuable correspondence with Jim Clunie.

\section{References}

1. W. Bergweiler, Iteration of meromorphic functions, Bull. Am. Math. Soc. 29 (1993), 151-188.

2. W. BergweILER, On the zeros of certain homogeneous differential polynomials, Archiv. Math. 64 (1995), 199-202.

3. W. BergWeiler AND A. EREMENKo, On the singularities of the inverse to a meromorphic function of finite order, Rev. Mat. Iberoamericana 11 (1995), 355-373.

4. A. Eremenko and M. Yu. Lyubich, Dynamical properties of some classes of entire functions, Ann. Inst. Fourier Grenoble 42 (1992), 989-1020.

5. G. FRANK, Eine Vermutung von Hayman über Nullstellen meromorpher Funktionen, Math. Zeit. 149 (1976), 29-36.

6. G. Frank and S. Hellerstein, On the meromorphic solutions of nonhomogeneous linear differential equations with polynomial coefficients, Proc. Lond. Math. Soc. (3) 53 (1986), 407-428.

7. G. Frank, W. Hennekemper and G. Polloczek, Über die Nullstellen meromorpher Funktionen and ihrer Ableitungen, Math. Ann. 225 (1977), 145-154.

8. W. K. Hayman, Picard values of meromorphic functions and their derivatives, Ann. Math. 70 (1959), 9-42.

9. W. K. Hayman, Meromorphic functions (Oxford, Clarendon, 1964).

10. W. K. HAYMAn, On the characteristic of functions meromorphic in the plane and of their integrals, Proc. Lond. Math. Soc. (3) 14A (1965), 93-128.

11. W. K. HAYMAn, The local growth of power series: a survey of the Wiman-Valiron method, Can. Math. Bull. 17 (1974), 317-358.

12. W. K. Hayman, Subharmonic functions, vol. 2 (London, Academic, 1989).

13. M. HECKNER, On meromorphic functions and linear differential polynomials which have few zeros, Mitt. Math. Sem. Giessen 211 (1992), 1-14.

14. E. Hille, Ordinary differential equations in the complex domain (Wiley, 1976). 
15. A. Hinkkanen, Reality of zeros of derivatives of meromorphic functions, Ann. Acad. Sci. Fenn. 22 (1997), 1-38.

16. A. Hinkkanen, Zeros of derivatives of strictly non-real meromorphic functions, Ann. Acad. Sci. Fenn. 22 (1997), 39-74.

17. F. R. KeOgh, A property of bounded Schlicht functions, J. Lond. Math. Soc. 29 (1954), 379-382.

18. J. K. LANGLey, Proof of a conjecture of Hayman concerning $f$ and $f^{\prime \prime}$, J. Lond. Math. Soc. (2) 48 (1993), 500-514.

19. J. K. LANGLEY, On second order linear differential polynomials, Resultate Mathematik 26 (1994), 51-82.

20. J. K. LAngley, On the multiple points of certain meromorphic functions, Proc. Am. Math. Soc. 123 (1995), 1787-1795.

21. J. K. LAngley, The zeros of the first two derivatives of a meromorphic function, Proc. Am. Math. Soc. 124 (1996), 2439-2441.

22. J. K. LAngley, On the zeros of the second derivative, Proc. R. Soc. Edinb. A 127 (1997), 359-368.

23. J. K. LANGLEY, On differential polynomials, fixpoints and critical values of meromorphic functions, Result. Math. 35 (1999), 284-309.

24. J. K. LAngley And D. F. SheA, On multiple points of meromorphic functions, J. Lond. Math. Soc. (2) 57 (1998), 371-384.

25. R. Nevanlinna, Eindeutige analytische Funktionen, 2nd edn (Springer, 1953).

26. G. PólyA, Über die Nullstellen sukzessiver Derivierten, Math. Z. 12 (1922), 36-60.

27. C. Pommerenke, Boundary behaviour of conformal maps, Grundlehren der Mathematischen Wissenschaften, vol. 299 (Springer, Berlin, 1992).

28. J. Rossi, Second order differential equations with transcendental coefficients, Proc. Am. Math. Soc. 97 (1986), 61-66.

29. T. Sheil-Small, On the zeros of derivatives of real entire functions and Wiman's conjecture, Ann. Math. 129 (1989), 179-183.

30. M. Tsuji, Potential theory in modern function theory (Tokyo, Maruzen, 1959).

31. G. VALIRON, Lectures on the general theory of integral functions (Toulouse, Edouard Privat, 1923). 